Maïté SNAUWAERT, «Fils du conte et de la fiction de soi : le roman de filiation québécois contemporain », dans Anne CAUMARTIN et Martine-Emmanuelle LAPOINTE (dir.), Filiations intellectuelles dans la littérature québécoise, @ nalyses, automne 2007

\title{
Maïté SNAUWAERT
}

\section{Fils du conte et de la fiction de soi : le roman de filiation québécois contemporain}

On a peu ou pas à ma connaissance remarqué l'incroyable parenté qui unit La Petite Fille qui aimait trop les allumettes de Gaëtan Soucy et Le Jour des corneilles de Jean-François Beauchemin, parus respectivement en 1998 (Boréal) et 2004 (Les Allusifs) ${ }^{1}$. De cette parenté je veux poser ici l'hypothèse qu'elle s'inscrirait dans le contexte plus large, repéré en France par Dominique Viart, de ce que celui-ci appelle les « récits de filiation » (1999, p. 117), qui me parait extensible au Québec contemporain. Ce contexte est celui d'un retour de la transivité du roman, qui se remet à raconter, à se donner des objets. Il est celui, également, d'une littérature dite postmoderne, dont Janet Paterson a montré les incidences majeures sur le roman : multiplication des voix narratives et éclatement du récit (p. 18-19). Enfin, ce contexte est celui d'une présence accrue des formes du biographique, si ce n'est d'une quasiexclusivité de ce qui est devenu l'un des genres ou modes majeurs de la littérature de la seconde moitié du $\mathrm{XX}^{\mathrm{e}}$ siècle et dont les explorations ne cessent de se poursuivre. Dans cette perspective, le roman contemporain, au Québec comme en France, est l'héritier et le continuateur d'une écriture en première personne qui rend apparentes et assume les marques de la subjectivité, sans que celle-ci soit nécessairement le gage d'une authenticité ou d'un «pacte » de vérité tel que le concevait Philippe Lejeune (p. 13).

Or je souhaite montrer comment les deux romans sélectionnés, en étant explicitement des fictions, en mettant en scène des univers litté-

\footnotetext{
1 Je n'en ai pas trouvé trace en tout cas dans la littérature critique, d'ailleurs inexistante pour Le Jour des corneilles, à peine plus fournie pour La Petite Fille qui aimait trop les allumettes, peut-être en raison de son grand succès populaire. Les deux livres, pourtant, ont reçu plusieurs prix : le prix France-Québec 2005 pour le premier, le prix Ringuet et le prix Grand Public La Presse pour le second.
} 
Maïté SNAUWAERT, «Fils du conte et de la fiction de soi : le roman de filiation québécois contemporain », dans Anne CAUMARTIN et Martine-Emmanuelle LAPOINTE (dir.), Filiations intellectuelles dans la littérature québécoise, @ nalyses, automne 2007

raires fortement codifiés, par le biais d'une langue savamment travaillée, l'emprunt d'une posture de conteur et des allusions à la littérature ou à l'apprentissage du langage, exhibent leur littérarité et, par là, le croisement entre leur héritage et leur inventivité, dans le même temps qu'ils se proposent de mettre en scène l'incidence de la filiation sur la construction d'une personnalité. Depuis une position initiale testamentaire se construit chez les deux narrateurs le processus d'une individuation, au sens d'une appropriation de soi (Ricœur, p. 86), opérée dans et par le langage, dans et par la langue singularisée en vecteur d'une diction de soi et, finalement, dans et par ce geste de conter qui évoque en dernier lieu la possibilité socialisante d'une transmission. Par là se fait jour, à mi-chemin du conte et de la fiction de soi, une sorte de nouvel avatar générique, Michel Biron parlant à propos de La Petite Fille... d'« une sorte de conte romanesque » (p. 407).

\section{Une position testamentaire}

Voyons d'abord quels moyens et modalités partagent les deux romans. En premier lieu, un narrateur (masculin chez Beauchemin, masculin puis féminin chez Soucy), très sommairement et tardivement nommé (respectivement le fils Courge et Alice), orphelin-e de mère de tout temps ou presque, de père depuis tout récemment, ce qui est l'élément déclencheur du récit. En outre, chacun de ces narrateurs se présente dans une situation de confession autobiographique, qui va dans le sens de l'aveu pour le fils Courge, bien que cet aveu — le parricide n'intervienne pour le lecteur qu'à l'issue du récit. Sa situation confessionnelle est celle de l'exposé devant un juge et un tribunal des circonstances qui l'ont mené là. Pour Alice, il s'agit davantage de consigner, au seuil d'un monde qui s'effondre, le souvenir de celui-ci pendant qu'il en est temps. Bertrand Gervais a montré à quel point le roman est marqué de cet imaginaire apocalyptique, de cette temporalité de «monde de la fin» (p. 386) qui précipite et oriente les gestes et leur signification. Mais à travers ceux de ce monde qui décline, Alice justifie également les raisons ou moyens de sa propre existence. Au demeurant, son discours, qu'elle appelle «testament», est tout aussi adressé que celui du fils Courge, ainsi qu'en témoignent les multiples mentions d'une seconde personne du pluriel et les allusions directes au 
Maïté SNAUWAERT, «Fils du conte et de la fiction de soi : le roman de filiation québécois contemporain », dans Anne CAUMARTIN et Martine-Emmanuelle LAPOINTE (dir.), Filiations intellectuelles dans la littérature québécoise, @ nalyses, automne 2007

lecteur futur de ces lignes. L'un et l'autre sont donc dans une position testamentaire par laquelle il s'agit à la fois de se justifier de ses propres agissements, d'en montrer les motivations et les conséquences potentielles ou, plus encore, étant donné qu'ils ne seront peut-être plus là pour les voir, l'enseignement qu'ils en ont tiré et qu'ils souhaitent euxmêmes transmettre. Ils sont ainsi dans l'exacte position d'héritiers à la charnière entre un legs reçu et un legs à déposer. Quoique l'un et l'autre soient dans une situation historique limite, marquée du sceau de la destruction ou de la perte — le fils Courge ne souhaite, à l'issue de cette confession et du jugement qui va s'ensuivre, qu'être exécuté, afin de rejoindre dans la mort ses parents —, il en va autrement de leur situation interne.

Le souhait du fils Courge rend compte de la grande sérénité qui lui est venue de son parcours : arrêté pour le meurtre de son père et emprisonné, c'est dans l'incarcération qu'il a connu, enfin, l'apprentissage du langage et des lettres qui, dans les enseignements de son père, lui avait tant fait défaut. À présent apaisé envers son père, envers lui-même et le monde, ayant perdu tous ceux qu'il aime (sa mère le jour de sa naissance, la femme dont il est tombé amoureux et qu'il n'a vue qu'un jour, son père, qu'il a tué et dépecé pour chercher en lui l'emplacement de l'amour qu'il ne lui rendait pas visible), il peut considérer d'un œil serein la perspective du trépas. Tandis qu'Alice, au terme du roman, est sur le point de donner la vie, d'enfanter une autre fille des œuvres de... son frère. Et bien qu'elle ait perdu elle aussi entre-temps l'homme étranger pour qui son cœur battait, elle accueille très favorablement et avec impatience cette naissance à venir, comme si elle devait inaugurer une nouvelle ère.

Aussi, bien que les tabous les plus extrêmes soient mis en œuvre dans les deux romans, n'irai-je pas dans le sens d'une hypothèse résolument négative ou encore moins pessimiste concernant l'issue et la signification de ces romans, mais plutôt dans celui de l'exposé d'un apprentissage de vie qui s'est fait, pour chacun de ces dépositaires, de façon finalement autonome, hors de l'autorité abusive d'un père qui, de part et d'autre, a surtout enseigné la violence. Transmise à son seul fils dans La Petite Fille..., transmise en partie à son fils dans le cas de 
Maïté SNAUWAERT, «Fils du conte et de la fiction de soi : le roman de filiation québécois contemporain », dans Anne CAUMARTIN et Martine-Emmanuelle LAPOINTE (dir.), Filiations intellectuelles dans la littérature québécoise, @ nalyses, automne 2007

Courge, quoique cette «violence » dont il fait preuve à l'encontre de son père ne soit ni barbare ni issue d'un accès de colère, mais consécutive à une décision, dont l'enjeu est vertueux ou, en tout cas, éminemment humain : chercher à «voir » l'amour, à en avoir le cœur net, si l'on peut dire, du fonctionnement de ce cœur de père, jusqu'où il a fallu percer, afin de le percer, littéralement, à jour. Avec les rudiments matériels — et tant soit peu parfois spirituels, mais totalement opaques et incompris des enfants - nécessaires ni plus ni moins qu'à la survie, chacun de ces pères a donné de par son propre modèle une figure d'autorité soit définitivement impossible à contrer (de sorte que, chez Soucy, le père est considéré comme un quasi-Dieu), soit susceptible d'être transgressée par le seul parricide. Malgré ces conditions délétères, se présentent deux voix qui entreprennent respectivement de se livrer à l'élaboration - rétrospective par définition - de leur identité narrative. Celle-ci doit être perçue positivement, en tant que construction d'un discours de soi sur soi, réinterprétation critique du donné et, partant, invention identitaire capable de se défaire du joug de l'autorité paternelle, voire d'une autre soumission masculine, celle du frère dans le cas d'Alice, qui a commis l'inceste. Cette reprise critique se montre ainsi à même de remettre chacun à sa place dans la généalogie, afin - et c'est le bénéfice ultime de ce travail d'émancipation - de se rendre soi-même responsable en vue d'une nouvelle filiation, soit directe, biologique et affective, pour Alice, soit indirecte pour le fils Courge, dont le caractère testamentaire du discours laisse à penser qu'il ne veut pas expier sa faute ni seulement l'expliquer, mais aussi faire de son histoire un exemple, qui puisse servir et devenir à son tour un récit qu'on transmet.

Ce qui rejoint l'hypothèse apocalyptique, sans doute allégorique d'ailleurs par rapport au monde contemporain et à ce fameux changement de millénaire, c'est bien que l'un et l'autre sont au seuil d'un changement de paradigme. Fût-ce dans le sang ou les éclairs et la fumée, Alice et le fils Courge s'apprêtent à quitter un monde d'obscurantisme, et ils sont les initiateurs de ce changement. Si la mort du père de la première intervient de soi-même, c'est pourtant Alice qui, responsable et consciente de devoir agir, commence par consigner celle-ci dans son carnet de "secrétarien », faisant le compte des événements quotidiens, 
Maïté SNAUWAERT, «Fils du conte et de la fiction de soi : le roman de filiation québécois contemporain », dans Anne CAUMARTIN et Martine-Emmanuelle LAPOINTE (dir.), Filiations intellectuelles dans la littérature québécoise, @ nalyses, automne 2007

avant de se rendre au village pour chercher un cercueil et pouvoir procéder aux rites funéraires et, donc, accomplir le changement. Elle est plus intelligente que son frère, mais aussi plus subtile que son père, et pour avoir beaucoup lu, n'ignore pas qu'il est des comportements humains élémentaires à observer en de telles circonstances. N'ayant en revanche jamais reçu d'affection de leur père, ni larmes ni autres formes d'émotion ne leur viennent naturellement. On retrouve également chez le fils Courge ce goût subit, dès que l'occasion lui en est donnée, pour la lecture et l'inventivité langagière, celle-ci étant le gage, non pas seulement d'une imagination féconde et d'une connaissance ici très biaisée et incomplète, mais surtout d'une grande faculté d'interprétation, donc d'une d'intelligence avec le monde et d'un très grand intérêt pour la communication - le langage étant, comme disait Benveniste, la communication (voir p. 258-266). D'une autre manière, le fils Courge manifeste tout au long des sentiments pour son père, mais puisqu'ils ne sont à ce point pas payés de retour et qu'au contraire, seuls le mutisme ou la violence gratuite de celui-ci s'exercent à son endroit, il se fait l'instigateur concret de cette sortie du noir.

\section{La langue comme relation}

Ce thème de l'obscurantisme est majeur dans les deux romans, d'abord représenté par l'absence totale de datation et de situation géographique (même si un village est nommé dans La Petite Fille...: "Saint-Aldor»), et plus encore par l'isolement de chacun des deux domaines, qui sont à l'écart du village et en totale autarcie, les deux pères n'entretenant, par un choix délibéré dont on ignore jusqu'à un certain point le secret, aucune relation avec les villageois, voire, chez Courge, avec l'ensemble de ses semblables. Cet obscurantisme s'exprime ensuite par le mutisme des deux pères, qui précisément ne livrent jamais les raisons de rien à leur progéniture et taisent absolument les conditions de l'enfance de celle-ci et les prémisses de l'histoire familiale. C'est ce sceau du secret qui, peut-on supposer, va rendre d'autant plus éperdue pour ces deux narrateurs la quête du sens et du récit de leurs origines, et donner lieu à l'invention d'un usage singulier de la langue, comme on le verra plus loin. Usage singulier de la langue qui la délie comme le moyen, justement, d'une relation perpé- 
Maïté SNAUWAERT, «Fils du conte et de la fiction de soi : le roman de filiation québécois contemporain », dans Anne CAUMARTIN et Martine-Emmanuelle LAPOINTE (dir.), Filiations intellectuelles dans la littérature québécoise, @ nalyses, automne 2007

tuelle, au double sens d'un récit et d'un lien. Dominique Viart écrit à propos du travail de la langue dans le roman contemporain :

Mais bien conscient [sic] que le sujet ne se trouve que dans la langue, et qu'il est à la fois révélé par elle en même temps que déformé et trahi par elle, l'écriture contemporaine s'installe dans cette conviction que l'épreuve de la langue est une épreuve du sujet. Une telle certitude exclut bien toute velléité d'«écrire comme », tout « retour à »: les formes académiques en effet ne se peuvent concevoir qu'en tant qu'elles sont entièrement dissociées d'une préoccupation du sujet-dans-la-langue, car la langue qu'elles offrent au sujet, toute normalisée par ses codes esthétiques, n'est pas propre à le dire. [...] Chaque sujet en effet se configure dans l'économie même de sa langue au moins autant sinon plus que dans le propos qu'il tient ou la fiction qu'il met en œuvre. (1999, p. 134)

Constat pertinent pour nos deux narrateurs, à cette différence près qu'ils investissent la langue, en particulier le fils Courge, d'une confiance sans réserve, comme si elle était en elle-même porteuse d'avenir, puisqu'elle a produit déjà leur émancipation. Et si Alice se montre peut-être plus critique, sceptique ou dubitative, c'est qu'elle reconnaît les propres failles de son éducation, mais jamais elle ne mettrait en doute les savoirs et la beauté dispensés par les livres. La fausse ignorance des pères, qui se manifeste d'abord par un refus de communiquer et d'être généreux, se trouve ainsi contrebalancée totalement par la langue très fleurie et ornementée des fils/fille qui, comme écrit Michèle Gazier à propos de La Petite Fille..., «fleure bon son XVIII ${ }^{\mathrm{e}}$ siècle $»^{2}$, autant dire celui des Lumières et de la culture lettrée qu'affectionnent tant Alice et Courge. Si d'ailleurs celui-ci, dont l'état civil est donné non pas même par son géniteur, mais par les gens du village lors de leur première rencontre, donc par la seule et première altérité terrestre et parlante (car le fils Courge rencontre bien des fantômes, mais ils sont muets), si donc son identité se résume à être le fils de son père, ce patronyme personnifiant l'inanimé légume tend à le rapprocher du conte, tandis qu'Alice, sans patronyme, mais avec un tel prénom, est fille du conte ou du moins de l'histoire pour enfants, comme aussi par le titre du roman, même si ce n'est pas elle qu'il dénomme. Il désigne cependant sa sœur jumelle, son autre littéraire, celle

${ }^{2}$ Michèle Gazier, «Maudit Québec », Télérama, n 2564, 3 mars 1999, p. 52; citée par Aurélien Boivin (p. 93). 
Maïté SNAUWAERT, «Fils du conte et de la fiction de soi : le roman de filiation québécois contemporain », dans Anne CAUMARTIN et Martine-Emmanuelle LAPOINTE (dir.), Filiations intellectuelles dans la littérature québécoise, @ nalyses, automne 2007

qui a, sinon péri, du moins succombé par les flammes, son imprudence punie, qui a tué leur mère et déclenché tout ce drame.

L'indétermination temporelle et géographique, de ce point de vue, alliée au thème des mauvais parents dont les enfants doivent se dégager, joue fortement de l'univers du conte, quoiqu'il s'agisse d'un conte moderne rendu possible par le fait d'avoir préservé à l'écart de la civilisation urbaine ces demeures d'un autre temps. Si Le Jour des corneilles est moins explicite, La Petite Fille... fait mention d'une moto chevauchée par l'inspecteur des mines, qui suffit à indiquer que le village, lui, est rendu à notre époque "moderne». L'aspect non merveilleux et, par conséquent, déceptif aux yeux d'Alice de l'architecture et de l'allure globale de ce village indique peut-être qu'il aurait plutôt revêtu la grisaille des villes. Dans l'autre roman, sans qu'on rencontre de détails aussi anecdotiques qu'un objet comme le moyen de transport motorisé, c'est bien sûr la possibilité d'apprendre en prison qui vient nous donner un facteur de situation dans l'Histoire. Les deux héritiers quittent un monde moyenâgeux (la noirceur sans le chevaleresque du médiéval) pour aborder tout à coup, grâce à la mort du père, dans le monde moderne d'une civilisation lettrée et dotée d'institutions. La déprise, cependant, des éléments géographiques et historiques, dont l'indétermination est dominante, renforce l'aspect explicitement littéraire de ces univers fictifs construits en référence au conte. Il convient d'examiner de plus près ces rapports.

\section{Dans le lignage du conte}

Chez Beauchemin, le « jour des corneilles » est le jour d'une première catastrophe qui a joué comme mauvais présage : celui de l'incendie qui a emporté en même temps les parents du père Courge et ceux de sa femme, ouvrant à une ère orpheline inconsolable, redoublée lorsque la mère du fils Courge meurt en lui donnant le jour. Jour singulier et fondateur, il a dans le texte la valeur de l'embrayeur des contes - Un jour ou il était une fois - et trouve son écho dans l'implicite jour du jugement dernier qui est l'aboutissement du récit en même temps que celui-ci tout entier le performe. La présence du surnaturel, enfin, sous la forme de personnages (les revenants que le fils Courge croise régulièrement sur 
Maïté SNAUWAERT, «Fils du conte et de la fiction de soi : le roman de filiation québécois contemporain », dans Anne CAUMARTIN et Martine-Emmanuelle LAPOINTE (dir.), Filiations intellectuelles dans la littérature québécoise, @ nalyses, automne 2007

son chemin, dont il est conscient qu'il bénéficie du « don » de les voir, non partagé par son père), sous la forme d'animaux qui souvent agissent ou d'impossibilités spatiotemporelles pourtant réalisées (le narrateur prétend se rappeler sa naissance ou affirme avoir passé plusieurs heures sous terre), apparente aussi bien le récit à la fable et contribue à doter le protagoniste de pouvoirs singuliers. Mais c'est surtout la vertu morale dégagée à la fin du récit qui vient achever de l'apparenter au conte, non seulement au plan formel, mais pour la place que celui-ci occupe dans l'histoire littéraire et dans l'imaginaire. Conformément au bénéfice produit par le conte tel que Bruno Bettelheim l'a mis en évidence dans La Psychanalyse des contes de fées (p. 35-36), il est possible de retirer une signification allégorique édifiante, appuyée sur un exemple concret dans lequel, s'il n'est pas possible de s'identifier directement, on pourra toujours puiser indirectement les enjeux nécessaires à notre projection et, par la suite, s'en servir sous ses différentes facettes, le redire en mettant en lumière tel ou tel de ses différents aspects' De façon somme toute binaire, la violence, expérimentée pour en découvrir l'inutilité, est rejetée au profit de la communication qu'est l'amour, qui ne peut que passer d'abord par la communication verbale, essentielle à la construction mimétique de l'humain comme être de langage, qui le distingue de l'animal. Cette simplicité de l'équation finale, qui n'est pas un simplisme puisqu'elle a fait l'objet d'un cheminement que retrace le récit, rejoint la valeur universelle du conte, dont le déploiement exige des situations exemplaires souvent extrêmes, dont il y aura à tirer la valeur, éventuellement différente pour chacun.

Chez Gaëtan Soucy, c'est dès le titre que s'affiche la référence au conte. Écrit par Andersen, La Petite Fille aux allumettes raconte l'histoire tragique d'une petite vendeuse qui, dans le froid et la faim d'une veille de Nouvel An, n'ose pas rentrer chez elle de peur de se faire frapper par son père, car elle n'a vendu aucune allumette. Orpheline de mère (dont il n'est pas du tout question) ainsi que de grand-mère, elle rêve de rejoindre celle-ci qui l'a un temps soignée, et, par un délire hallucinatoire de la faim, la voit apparaître dans la lumière que jettent les pauvres flammes obtenues pour se réchauffer à la faveur de quelques allumettes craquées. Poursuivant sa vision, elle met bientôt en cendres 
Maïté SNAUWAERT, «Fils du conte et de la fiction de soi : le roman de filiation québécois contemporain », dans Anne CAUMARTIN et Martine-Emmanuelle LAPOINTE (dir.), Filiations intellectuelles dans la littérature québécoise, @ nalyses, automne 2007

toute sa provision et est retrouvée morte gelée le lendemain matin, jour d'une nouvelle année. L'ambivalence de ce conte est grande, étant donné que la mort, ici, qui lui fait rejoindre une figure aimée, est sans doute une issue meilleure que la survivance misérable et le retour pénible dans une chaumière qui n'est chauffée ni par le bois ni par l'amour ou le travail du père. Pourtant, elle joue aussi comme punition de la transgression de l'interdit concernant le jeu avec le feu et, dans le roman de Soucy, dont le titre met l'accent sur un comportement jugé déviant («qui aimait trop les allumettes»), on imagine sans peine, commençant en quelque sorte par la fin, que du mauvais va sortir de ce vice. C'est en fait déjà fait et nous n'assistons d'abord qu'aux conséquences avant de remonter lentement vers les causes. La narration, en fait, se déploie dans un temps qui, de ce point de vue, est postcatastrophique, comme l'a remarqué Bertrand Gervais (p. 389). Le drame a été joué, et l'histoire se construit sur ses ruines. Mais ce qu'elle construit à son tour, ce vers quoi elle s'oriente, ce ne sont pas des ruines ni de l'éphémère ni du feu; c'est bien une vie nouvelle et une nouvelle filiation, marquée de l'alternative du féminin : «Oui je dis elle car ce sera une angelote à qui je serai une goutte d'eau, j'en veux pour preuve la conviction que je sens dans mon ventre. » (2000 [1998], p. 178) Qui en tant que telle s'annonce capable de s'y prendre autrement en matière d'éducation: "Elle grandira sans horion aucun, comme les fleurs qui n'ont pas besoin qu'on les maltraite pour pousser toutes couleurs dehors. Elle sera attentive et polie à l'égard des bêtes, elle ne les abandonnera pas dans le désemparement et la famine, comme hélas j'en connais, qui grilleront. » Mais capable aussi de réparer, en évitant de les reproduire, les erreurs du passé, d'en tirer, pour qu'elles n'aient pas été vaines, un enseignement: "Je lui apprendrai enfin à se méfier comme du feu des poupées séductrices et ravageuses, dangereuses à force de beauté, car selon les dictons de mon père, c'est à quatre ans qu'on aime trop les allumettes, et je l'appellerai Ariane, en mémoire du châtiment...» (p. 178-179). Il s'agira donc d'une autre fille mythologique, d'une généalogie moins biologique que littéraire et mémorielle, dont le baptême s'emploiera à perpétuer une mémoire, à entériner et à dire une histoire au lieu de la recouvrir et de l'escamoter. Or Jean-Noël Pelen, dans sa réflexion sur la réitération du conte, dégage une autre valeur qui n'est pas la morale traditionnelle, ou encore 
Maïté SNAUWAERT, «Fils du conte et de la fiction de soi : le roman de filiation québécois contemporain », dans Anne CAUMARTIN et Martine-Emmanuelle LAPOINTE (dir.), Filiations intellectuelles dans la littérature québécoise, @ nalyses, automne 2007

la vertu éducative, mais le bénéfice triple de sa nature narrative : d'une part « [1] conformité réitérée du conte crée un îlot de mémoire primitive, dans lequel l'individu se reconnaît tout au long de sa vie ", d'autre part il s'agit « d'une conformité créatrice, qui ne connait pas la contingence du sens concret, car la forme est le sens. De ce point de vue-là, il s'agit d'un acte [...] mythique, qui vérifie la puissance créatrice du Verbe.» Enfin le conte, comme toute création, «ordonne» (p. 212). La qualité que Pelen propose comme «définitoire du conte» est ainsi «la (re)production de la conformité narrative » (p. 210). Cependant, la narration étant, dans nos deux exemples, à ce point singularisée, autant au sens de l'originalité qu'à celui, conséquent, d'une occurrence unique, la conclusion à en tirer pour ce qui nous concerne irait davantage dans le sens de la production de la conformité narrative : ouvrant par et dans un langage qui n'a pas été transmis, mais acquis, la voie d'une filiation nouvelle, les narrateurs inaugurent à proprement parler un régime de la parole qui ne se veut pas fictionnel, mais référentiel, articulé à l'expérience et capable d'en rendre compte comme de s'en dégager, fondant par là de toutes pièces un monde nouveau, harmonisé par cet usage de la communication qui met en ordre et relie, à commencer par l'histoire de sa propre vie et son «identité narrative ».

C'est ainsi surtout la posture dans le langage qui est celle du conteur au sens fort : d'une voix à la fois fortement singulière et à visée collective, en position de transmission entre un monde révolu et un monde à venir. Le degré maximal d'accomplissement, selon Walter Benjamin, étant le conte puisé dans une expérience personnelle, particulière et concrète, dont est tiré un savoir déjà éprouvé et donc assurément valide pour la communauté qui en est instruite. "Tous les grands conteurs ont [...] en commun l'aisance avec laquelle ils montent et descendent les échelons de leur expérience comme ceux d'une échelle. Une échelle qui s'enfonce dans les entrailles de la terre, et qui se perd dans les nuages » (p. 140), écrit Benjamin dans l'essai éponyme. La qualité première du conteur est alors la façon dont il fait corps avec son récit, pour convaincre de sa valeur empirique et le rendre vivant, ainsi suffisamment attractif et mémorable pour ses auditeurs. Vertu que s'octroient les deux textes par leur usage prégnant de la première personne, leur personnalisation très littéraire de la langue et leur dimension forte d'adresse, qui entérinent leur position 
Maïté SNAUWAERT, «Fils du conte et de la fiction de soi : le roman de filiation québécois contemporain », dans Anne CAUMARTIN et Martine-Emmanuelle LAPOINTE (dir.), Filiations intellectuelles dans la littérature québécoise, @ nalyses, automne 2007

symbolique de référents langagiers. Enfin, la tension de leur énonciation entre un présent de relation et un passé (simple ou composé) de récit rejoint le constat de Dominique Viart selon lequel les récits contemporains renouent avec le souci de raconter tout en étant les dignes fils de l'ère critique du soupçon et confirme donc l'historicité singulière de leur position d'héritiers : «Le récit qui nous est revenu est un récit présent, fût-il un récit de mémoire. [...] il met l'accent sur la discordance qui se creuse entre la position du narrateur et la matière de sa narration. Le récit est ainsi devenu une forme toujours en question, en déséquilibre.» (1998, p. 26) De fait, chacune des instances narratives étudiées problématise en les circonstanciant les conditions de sa mise en récit, ne serait-ce que par le moment-limite où elle se situe. On lit ainsi au dernier paragraphe de l'incipit en forme de prologue du Jour des corneilles:

Car s'il me faut aujourd'bui tourner pour vous les pages de mon existence, il me faudra aussi, par même occasion et pour mieux traduire mon récit, ouvrir le livre de la vie de père, si étroitement emmaillotée à la mienne. Cela afin de vous instruire meilleurement des circonstances où je fus conduit à achever mon prochain, puis enseigné de vocabulaire et, enfin, mené ci-devant vous et les membres de ce tribuneau pour trancher mon cas. (p. 9)

Tandis que La Petite Fille... commence en ces termes : «Nous avons dû prendre l'univers en main mon frère et moi car un matin peu avant l'aube papa rendit l'âme sans crier gare.» (p. 13) Ce qui réalise le mieux la synthèse entre tradition et inauguration, ce qui fait le mieux entendre cette discordance entre position du narrateur et matière de sa narration, c'est alors l'investissement idiosyncrasique d'une langue qui est toujours à tous et déjà existante, mais qui découvre des sonorités nouvelles et peut-être inédites lorsqu'elle est rendue étrangère par un usage décalé. L'appropriation, alors, de l'hérité, du transmis, qui s'incarne dans la langue comme code, comme convention de communication, se fait par l'accentuation de l'impropriété du linguistique, qui en réalise le dépaysement interne.

\section{Des langues de l'individuation}

Tournures très ampoulées du fils Courge («Monsieur le juge », « Père »), ou familières d'Alice ( majuscule, comme plus loin « spinoza ») : dans les deux cas, la langue est 
Maïté SNAUWAERT, «Fils du conte et de la fiction de soi : le roman de filiation québécois contemporain », dans Anne CAUMARTIN et Martine-Emmanuelle LAPOINTE (dir.), Filiations intellectuelles dans la littérature québécoise, @ nalyses, automne 2007

inventive, personnalisée par le narrateur qui la déroule autant que son récit et semble l'inventer à mesure. Chez le premier, c'est aussi lexicalement qu'elle se fait idiosyncrasique, notamment par l'inversion ou la substitution de suffixes, notamment entre le féminin et le masculin, ou l'adjectif et le nom, ou encore la formation de noms ou d'adverbes à partir d'un adjectif, qui créent des semi-néologismes précieux, ampoulés, fortement littéraires : «tribuneau» (p. 9), «vertes musclures » (p. 19), «terribleries» (p. 19), «trembleuses» (p. 19), « chérissement» (p. 67), etc. Invention qui donne l'impression d'une langue d'exception, propre, comme le relevait Dominique Viart, à dire le sujet dans toute sa singularité, mais aussi à donner un tour très littéraire à la manière du conteur, qui enflamme son histoire en lui conférant la patine de faux archaïsmes alliée à la poésie impromptue de mots techniques contemporains, dans une tournure qui cumule figures et exclamations pour tirer du monde concret une valeur allégorique :

Un soir, nous étions affairés à bourrer de polytric le fondement de nos godillots. Les insectes, ces petits ménestrels de la nuit, emplissaient l'air de leurs chants obstinés. Des mouches-de-feu passaient. Tandis que je guettais l'embrasement de leurs ventres extravagants, je goûtais semblablement un feu en moi-même. [...] Surprise des jours! Lors même que le malheur s'abat sur nous comme grêlons, voici que brille en nos ventres une joie, courtaude et ténue, mais pourtant suffisante pour pardonner au sort ses rudesses! (Beauchemin, p. 66)

Chez Alice, c'est surtout par la franche liberté avec laquelle elle s'en saisit que la langue lui devient familière au double sens du terme : porteuse d'affects d'abord, qui sont des jugements ou des critiques aussi bien que des sentiments, mais font voir à tout moment la position qui est la sienne en tant que locuteur; ramenée, ensuite, à sa mesure individuelle, située, subjective : conjuguée au masculin dans la première partie, au féminin dans la seconde, et adaptée à son point de vue et à sa connaissance du monde en tant qu'enfant. La mise en œuvre d'une langue nouvelle est également prégnante, mais principalement par l'usage détourné des formules, qui conduit en les littéralisant à les sortir de leur fixité et à les remettre dans une circulation vivante, montrant par là un travail d'appropriation. Les expressions toutes faites qui lui sont adressées sont prises au premier degré par la narratrice, ce qui leur donne une autre couleur, en même temps que des 
Maïté SNAUWAERT, «Fils du conte et de la fiction de soi : le roman de filiation québécois contemporain », dans Anne CAUMARTIN et Martine-Emmanuelle LAPOINTE (dir.), Filiations intellectuelles dans la littérature québécoise, @ nalyses, automne 2007

façons de parler très contemporaines sont intégrées dans un récit ampoulé qui ne les appelait pas, contrastant avec ce ton et avec la gravité des événements rapportés, ce qui contribue, avec l'autodérision qui caractérise son énonciation rapide, au comique de l'éloquence d'Alice :

J'avais bien sûr un peu menti au poète des mines quand je vous avais dit que je n’avais jamais pleuré de ma putain, parce qu'il y avait bien les fois où papa nous faisait l'attacher aux chaînes des portes de la galerie de portraits et qu'il me forçait à le frapper avec un linge trempé, [...] mais j'avais dit cela à l'inspecteur pour faire mon indépendante et montrer que j'avais ma dignité aussi, pour qu'il me trouve fascinante ainsi qu'intensément jolie. (2000 [1998], p. 153)

Fortement déictique enfin, cette écriture destinée à accomplir la mission de « secrétarien » opère en réalité l'individuation de son auteure, à qui elle bénéficie en dernière analyse (ou peut-être à sa fille?), même si le détour de l'adresse est nécessaire, comme est nécessaire, dans toute filiation, que s'accomplisse le processus d'une transmission. Alice, à l'issue de son récit, s'est émancipée de son père et de son frère, a conquis sa sexuation qui ne lui était pas reconnue et s'est vue dans les yeux d'un autre qui s'est épris d'elle. Elle a trouvé à se séparer de l'informe organisation familiale qui ne la spécifiait pas comme individu, mais la résumait à une fonction relative et un rôle assujetti et, sans se soumettre à un ordre autre que le sien, elle s'apprête comme le fils Courge à assumer une vie responsable et autonome, quoiqu'en relation avec sa descendance.

\section{Des fictions du soi}

Ainsi, avec des moyens inverses, les textes parviennent au même effet secondaire de déréaliser les événements racontés par la prééminence de la subjectivité qui s'exprime à travers cette inventivité langagière. Il en résulte un modèle de narrateur à la fois cognitif et relationnel, qui semble ouvrir la voie d'un schéma d'émancipation et, à tout le moins, d'individuation. Et ce, par une construction littéraire d'identité narrative dont tout un chacun semble pouvoir s'approprier les moyens. Ces narrateurs assument ainsi la médiation du langage, qui semble avoir pris la place entière des parents dans le rôle d'intermédiaire avec le monde, comme dans le rôle de l'apprentissage relationnel au sens 
Maïté SNAUWAERT, «Fils du conte et de la fiction de soi : le roman de filiation québécois contemporain », dans Anne CAUMARTIN et Martine-Emmanuelle LAPOINTE (dir.), Filiations intellectuelles dans la littérature québécoise, @ nalyses, automne 2007

large. Quoique solitaires, ils restaurent en écrivant le continuum d'une filiation qui a été brisée. C'est que tous deux, entre une mère absente et un père inexpressif, ont appris à parler dans les livres. Ils sont les produits littéraires de cette filiation par la lettre qui leur a donné ses modèles narratifs et langagiers et, pourrait-on dire, récitatifs: conjuguant sans cesse la parole à l'histoire, l'illocutoire à l'éloquence, faisant de la relation, à tout moment, autant un lien qu'un récit. Mais ils sont aussi, de ce fait, en quelque sorte fils de leurs propres œuvres : inaugurant un parler nouveau (une parole nouvelle?), à l'aube de l'apparition, sur les ruines de l'ancien, d'un monde dont ils se montrent, même s'ils n'y seront plus, même s'ils ne doivent pas le voir, préoccupés.

\section{Bibliographie}

BEAUCHEMIN, Jean-François. 2004, Le Jour des corneilles, Montréal, Les Allusifs.

Benjamin, Walter. 2000 [1936], «Le conteur. Réflexions sur l'œuvre de Nicolas Leskov », dans Euvres III, Paris, Gallimard, coll. «Folio Essais », ch. 4.

BENVENISTE, Émile. 1966, «De la subjectivité dans le langage », Problèmes de linguistique générale, t. I, Paris, Gallimard, coll. « Tel », p. 258-266.

Bettelheim, Bruno. 1979, La Psychanalyse des contes de fées, trad. Théo Carlier, Paris, Robert Laffont, coll. «Le Livre de poche ».

BIRON, Michel. 1999, «Autour de quelques morts », Voix et Images, vol. XXIV, $\mathrm{n}^{\circ} 2$, p. 407-412.

BoIVIN, Aurélien. 2001a, «La Petite Fille qui aimait trop les allumettes ou la métaphore du Québec », Québec français, n 122, p. 90-93.

GERVAIS, Bertrand. 2001b, «L'art de se brûler les doigts. L'imaginaire de la fin de La Petite Fille qui aimait trop les allumettes de Gaëtan 
Maïté SNAUWAERT, «Fils du conte et de la fiction de soi : le roman de filiation québécois contemporain », dans Anne CAUMARTIN et Martine-Emmanuelle LAPOINTE (dir.), Filiations intellectuelles dans la littérature québécoise, @ nalyses, automne 2007

Soucy », Voix et Images, vol. XXVI, n² 2 (77), p. 384-393.

LeJeune, Philippe. 1975, Le Pacte autobiographique, Paris, Seuil, coll. «Points ».

PATERSON, Janet M. 1990, Moments postmodernes dans le roman québécois, Ottawa, Presses de l'Université d'Ottawa.

PELEN, Jean-Noël. 2002, «Le simple fait de raconter toujours la même histoire : Réflexion sur l'en-deçà du sens dans la tradition du conte », dans André PETITAT (dir.), Contes : L'universel et le singulier, Lausanne, Payot, p. 197-213.

RICCeur, Paul. 2000, La Mémoire, l'histoire, l'oubli, Paris, Seuil.

Soucy, Gaëtan. 2000 [1998], La Petite Fille qui aimait trop les allumettes, Montréal, Boréal Compact.

VIART, Dominique. 1998, « Mémoires du récit. Questions à la modernité », dans Écritures contemporaines 1. Mémoires du récit, Paris-Caen, Minard, coll. «Lettres modernes », p. 3-27;

—. 1999, «Filiations littéraires », dans Jan BAETENS et Dominique VIART (dir.), Écritures contemporaines 2. États du roman contemporain, Actes du colloque de Calaceite, 6-13 juillet 1996, ParisCaen, Minard, coll. « Lettres modernes », p. 134-139. 